\title{
Inhaltsverzeichniss,
}

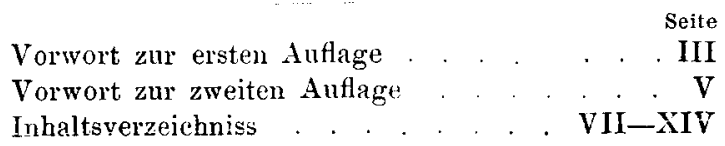

Einleitung.

\$. 1. Einleitung . . . . . . . . . . . . . . . 1

\$. 2. Definition von Krankheit . . . . . . . . . . 1

\$. 3. Symptome . . . . . . . . . . . . . . . . 1

\$. 4. Subjective Symptome . . . . . . . . . . 1

§. 5. Objective Symptome . . . . . . . . . . . 1

\$. 6. Pathognomonische Symptome . . . . . . . 1

\$. 7. Negativ-pathognomonische Symptome . . . . 2

\$. 8. Diagnose . . . . . . . . . . . . . . . 2

ร. 9. Krankenexamen , . . . . . . . . . . 2

§. 10. Objective Untersuchung . . . . . . . . . . . 2

\$. 11. Ausschliessungsdiagnose . . . . . . . . . 3

\$. 12. Prognose . . . . . . . . . . . . . . . 3

\$. 13. Dauer, Verlauf, Ausgänge . . . . . . . . 4

\$. 14. Wiederholung der Untersuchung . . . . . . 5

\$. 15. Section. . . . . . . . . . . . . . . . 5

\section{Allgemeiner Theil.}

\$. 16. Theile der Krankenuntersuchung . . . . . . 6

§. 17. Mittel zur Feststellung des Status praesens . . 6

§. 18-22. Inspection . . . . . . . . . . . . 6-10

\$. 23-26. Palpation . . . . . . . . . . . $10-15$

§. 27. Mensuration . . . . . . . . . . . . . 15

§. 28. Physikalische Untersuchung im engeren Sinne 15

\$. 29-36. Percussion . . . . . . . . . . 15-19

§. 37-59. Auscultation . . . . . . . . . . 19-28

§. 60-83. Thermometrie und Fieberlehre . . . $29-35$

§. 84 u.85. Expectoration . . . . . . . . . 36

§. 86. Haemorrhagieen .. . . . . . . . . . $37-39$

§. 87--96. Untersuchung des Urins. . ... . . 40-65

8. 87-89. Normale Harnbestaudtheile . . . $40-43$

8. 90. Abnorme Harnbestandtheile . . . . 43-45

§. 91-93. Harnsedimente . . . . . . . . 45-54 
$\mathrm{x}$

§. 94. Qualitative Bestimmung der laautsäch-

liebsten Bestandtheile des Urins . . 54-50

\$. 95. Kurze chemische Untersuchung . . . 56 u. 57

\$. 96. Specielle chemische Untersuchung . . 57-65

\section{Anweisung zur Anstellung einer allgemeinen klinisehen} Untersuchung.

\$. 97. Verhalten am Krankenbette. . . . . . . . 66

\$. 98. I) Anamnese . . . . . . . . . . . . 66;

\$. 99. Frforschung der ersten Symptome . . . . . 68

\$. 100. Erforschung der lokalen Erscheinungen . . . 67

\$. 101. II) Status praesens. Allgemeinzustand. . 69

\$. 102. Zustand des Nervensystems . . . . . . . $\quad 69$

\$. 103. Zustand der Musculatur . . . . . . . . . 70

s. 104. Zustand der Gelenke . . . . . . . . . . 70

§. 105. Zustand der Wirbelsäule. . . . . . . .

\$. 106. Zustand des Kopfes . . . . . . . . . . . . 70

\$. 107. Zustand des Halses . . . . . . . . . . . . . 71

\$. 108. Zustand des Herzens und anderer Allgemeinerscheinuugen der Circulation . . . . . it

\$. 109. Kustand der Respiration . . .. . . . . . . . 73

\$. 110. \%ustand der Haut in Allgemcinen . . . . . 73

\$. 111. Zustand des Digestionsapparates. . . . . . 73

a) Lippen . . . . . . . . . . . . . . 73

b) Zäbne . . . . . . . . . . . . . . . . 73

c) Zahnfleisch . . . . . . . . . . . . . . . 74

d) Mundsehleimhaut . . . . . . . . . . 74

e) Zunge . . . . . . . . . . . . . . . 74

f) Pharynx und lesophagus . . . . . . 75

g) Magen . . . . . . . . . . . . . . 75

li) Darmkanal . . . . . . . . . . . . . . . 76

\$. 112. Zustand des uropoëtischen Systems . . . . . 76

§. 113. Zustand der Brusthöhle und deren Organe . . 78

ร. 114 128. Die versehiedenen Regionen der Brust 78-81

§. 129. Lage des Herzens . . . . .. . . . . . . 81

\$. 130. Lage der Ostien und Klappen des Herzens . . 82

§. 131. Zustand der Unterleibshöble und deren Organe 85

\$. 132. 138. Die verschiedenen Regionen des Unterleibs . . . . . . . . . . . . $85-87$

§. 139. Allgemeinzustand des Unterleibs . . . . . . 87 
seite

3. 140. Obere Grenze ron Flitsjutreit in der Bauchböhle co

\$. 141. Unterschied der Zeichen bei Aseites und $\mathrm{Hy}$ drops ovarii . . . . . . . . . . . . . SS

§. 142. Lage der Leber . . . . . . . . . . . . . . 88

\$. 143. Lntersuchung der Iseber. . . . . . . . 8!

s. 144. Lage des Magens . . . . . . . . . . $\$ 1$

ॐ. 145. Untersuchung des Magens . . . . . . . . . 91

\$. 146 u. 147. Palpation des Magens . . . . . 91 u. 92

\$. 148. Palpation des Pylorus . . . . . . . . . . 92

今. 149. Untersuchung der Därme . . . . . . . . 9:3

\$. 150. Palpation der Dürme . . . . . . . . . . 93

\$. 151. Palpation und Percussion res Ilickdarms . . It

\$. 152. Untersuchung des Afters. . . . . . . . . 94

ॐ. 153. Lage der Milz . . . . . . . . . . . . . . . 94

\$. 154. Unitersuchung der Milz . . . . . . . . . . .5

§. 155. Lage des Pankreas . . . . . . . . . . 96

\$. 156. Untersuchung des Prankreas . . . . . . . 96

\$. 157. Lage und Untersuchung der Nieren . . . . 96

\$. 158. Lage und Untersuchung der Lreteren . . . . 96

§. 159. Lage und L'ntersuchung der ILamblase . . . 97

\section{Specielle klinisehe tutersuchung.}

1) Kranke mit Symptomen, welche auf eine Erkrankung der Lunge oder Pleura deuten.

§. 160 u. 161. Acute Brustkianklieiten . . . . . . 98

\$. 162. Chronische Brustkrankheiten . . . . . . 98

§. 163. Weg zur Diagnose . . . . . . . . . . . 98

§. 164. Bronchitis . . . . . . . . . . . . . 98

\$. 165. Pneumonie . . . . . . . . . . . . . . 99

\$. 166. Chronische Lungentuberculose. . . . . . . 99

s. 167. Pneumothorax . . . . . . . . . . . . . 100

\$. 168. Emphysem . . . . . . . . . . . . . 100

\$. 169. Pleuritis . . . . . . . . . . . . . . . 100

§. 170. Hydrothorax . . . . . . . . . . . . . 101

2) Krankemit Symptomen, welche a f eime Erkrankung des Herzens oder der grossen Gefässedeuten.

§. 171 u. 172. Fieberhafte Herzkrankheitcn . . . . . 101

§. 173. Fieberlose Herzkrankheiten . . . . . . . . 101

\$. 174. Weg zur Diagnose . . . . . . . . . . . 102 
8. 17. Aeute Pericarditis . " . - - 102

\$. 176. Acute Myocarditis . . . . . . . . 102

\$. 177. Acute Encarditis . . . . . . . . . . . 102

§. 178. Hydropericardium . . . . . . . . . . . 103

\$. 179. Chronische Hyocarditis . . . . . . . . . 103

\$. 180. Hypertrophieen des Herzens . . . . . . . 103

a) Excentrische Hypertrophie des linken Herzens 103

b) Excentrische Hypertrophie des rechten Herzens 103

§. 181. Herzatrophie . . . . . . . . . . . . . 103

\$. 182. Fettdegeneration cles Herzens. . . . . 104

§. 183. Organische Herzfehler . . . . . . . . . 104

1) Fehler der Valvula mitralis . . . . . . 104

a) Insufficienz der Yitralklappe . . . . . . 104

b) Sterose am linken Ostium venosum . . . 105

c) Stenose mit Insufficienz . . . . . . . . 105

2) Fehlẹ der Aortenklappen . . . . . . . 105

a) Iusufficienz der Aortenklapjen . . . . 105

b) Stenose am Ostium aorticam . . 105

c) Insufficienz und Stenose . . . . . 105

3) Fehler der Valvula tricuspidalis . . . . . . 105

a) Insufficienz der Tricuspilalklappe . . . . 105

b) Stenose am Ostiun venostun dextrmm , , 106

4) Febler der Pulmonalarterienklappen . . . 106

a) Insufficienz . . . . . . . . 106

b) Stenose am Ostiun der Pulmonalinterie . . 106

\$. 184. Aortenaneurysmen . . . . . . . . . . . 106

3) Kranke mit Symptomen, welche auf eine krkrankung eines Organs der Unterleibshöhle deuten.

§. 185. 1) Magenkrankheiten . . . . . . . . . . 107

§. 186. Acuter Magenkatarrl . . . . . . . . 107

\$. 187. Chronischer Magenkatarrlı . . . . . 107

§. 188. Perforirendes Magengesch wiil . . . . . 107

§. 189. Magenkrebs . . . . . . . . . 107

\$. 190. 2) Darmkanalkrankheiten . . . . . . . 107

§. 191. Acuter Dünndarmkatarrh . . . 108

§. 192. Chronischer Dïnndarmkatarrh . . . . . 108

s. 193. Perforirendes Duodenalgesehwïr . . . 108

\$. 194. Typhlytis und Perityphlitis _ 108

\$. 195. Proktitis und Periprolititis . . . . . 108 
\$. 196. Mastdarmkrebs . . . . . . . . . . 108

8. 197. Innere Einklemmung, Volvulus, Invagination 109

§. 198. Entozö̈n. . . . . . . . . . . . . 109

ॐ. 199. 3) Leber- und Gallenwegekrankheiten " . . . 110

§. 200. Allgemeine interstitielle fibröse Hepatitis

(Lebercirrhose, granulirte Leber). . . 110

§. 201. Suppurative Hepatitis (Leberabscess) . . 110

\$. 202. Acute gelbe Leberatrophie . . . . . . 110

8. 203. Leberkrebs . . . . . . . . . . . . 111

§. 204. Fettleber . . . . . . . . . . . 111

\$. 205. Echinococcus der Leber . . . . . . . 111

\$. 206. Thrombose der Pfortader . . . . . . 111

\$. 207. Pylephlebitis suppurativa. . . . . . . 112

§. 208. Hepatogener Icterus .. . . . . . . . . . 112

§. 209. Hämatogener Icterus . . . . . . . . . 112

§. 210. Gallensteine . . . . . . . . . . . . 113

§. 211. 4) Bauchfellkrankheiten . . . . . . . . . 113

\$. 212. Peritonitis . . . . . . . . . . . . 113

8. 213. Ascites . . . . . . . . . . . . 113

§. 214. 5) Milzkrankheiten . . . . . . . . . . 113

§. 215. Acuter Milatumor . . . . . . . . . 113

§. 216. Chronischer Milztumor . . . . . . . 114

\$. 217. 6) Nierenkrankheiten . . . . . . . . . . 114

§. 218. Stauungshyperämie . . . . . . . . . 114

§. 219. Desquamative Nephritis . . . . . . . 114

\$. 220. Parenchymatöse Nephritis . . . . . . 115

§. 221. Cluronischer Morbus Brightii . . . . . 115

8. 222. Amyloide Degeneration d. Niere (Speckniere) 115

§. 223. Abnorme Beweglichkeit und Lageveränderung der Niere. . . . . . . . . . 116

§. 224. Addison'sche Krankheit . . . . . . . 116

4) Die Hautkrankheiten.

§. 225. Acute und chronische Hautkrankheiten . . . 116

§. 226. Grundformen . . . . . . . . . . . . . 116

§. 227. Acute contagiöse Exantheme . . . . . . . 116

§. 228. Acute nicht contagiöse Exantheme . . . . . 116

§. 229. Chronische Hautkrankheiten . . . . . . . 117

§. 230. Fernere Eintheilung der Hautkrankheiten . . 117 
5) Kranke mit Symptomen, welche tuf eine Erkrankung des Gehirns oder seiner Häute deuten.

§. 231. Krankheiten des Gehirns und der Himbiute. 119

§. 232. Acute und chronische Gebirn- und Hirnhautkrankbeiten . . . . . . . . . . . 120

§. 233. Functionelle Störungen . . . . . . . . . 120

\$. 234. Sensible Reizungserscheinungen. . . . . . 120

\$. 235. Sensible Depressionserscheinungen . . . . . 120

\$. 236. Motorische Reizungserscheinungen . . . . . 120

\$. 237. Motorische Depressionserscheinungen . . . 120

\$. 238. Reizung des Vagus . . . . . . . . . 120

\$. 239. Lähmung des Vagus . . . . . . . . . . . . 120

§. 240. Psychische Reizungserscheinungen . . . . 120

\$. 241. Psychische Depressionserscheinungen . . . 121

\$. 242. Gruppen von Functionsstörungen . . . . . 121

§. 243. Erscheinungen von Affectionen der Hirnconvexität, der Hirnganglien u. der Grosshirnschenkel 121

ร. 244. Hemiplegische Affectionen . . . . . . . . 121

§. 245. Erscheinungen bei Erkrankungen der Fossae Sylvii . . . . . . . . . . . . . . . 121

૬. 246. Erscheinungen von Affectionen der Hirnbasis 121

\$. 247. Einseitige Hirnerklankung . . . . . . . 121

§. 248. Functionsstörungen beider Körperbältten , . 121

\$. 249. Herdsymptome . . . . . . . . . . . 121

\$. 250. Meningitis der Convexität . . . . . . 122

\$. 251. Meningitis dex Basis . . . . . . . . . 122

\$. 252. Epidemische Cerebrospinalmeningitis . . . . 122

\$. 253. Apoplexia cerebri . . . . . . . . . . . 123

§. 254. Embolie der Arteria fossae Sylvii . . . . . 123

§. 255. Hirn- und Hirnhauttumoren . . . . . . . 124

§. 256. Encephalitis (Hirnabscess) . . . . . . . . 125

\$. 257. Chronischer Hydrocephalus . . . . . . . 125

6) Kranke mit Symptomen, welche auf eine Erkrankung des Rückenmarks und seiner Häute deuten.

§. 258. Krankheiten des Rückenmarks und seiner Häute 125

§. 259. Fieberhafte Rückenmarkskrankheiten . . . 126

\$. 260. Eintheilung der Symptome. . . . . . . . 126

§. 261. Motorische Reizungserscheinungen . . . . . 126 
\$. 262. Motorische Depressionserscheinungen . . . . 126

§. 263. Sensible Reizungserscheinungen . . . . . . 126

§. 264. Sensible Depressionserscheinungen . . . . 126

ร. 265. Coordinationsstörungen . . . . . . . . . 126

५. 266. Paraplegische Affectionen . . . . 126

§. 267. Meningitis spinalis acuta . . . 127

§. 268. Meningitis spinalis chronici. . . 127

§. 269. Myelitis acuta . . . . . . . . . . . 127

\$. 270. Myelitis chronica . . . . . . . . . . 127

\$. 271. Tabes dorsualis . . . . . . . 128

7) Kranke mit Symptomen, welche ant eine Erkrankung der peripheren Nerven deaten.

\$. 272. Krankheiten der peripheren Nerven . . . . 128

\$. 273. Tic convulsif . . . . . . . . . . 128

\$. 274. Hemikranie . . . . . . . . . . . . . 128

8) Allgemeine Neurosen.

\$. 275. Allgemeine Neurosen . . . . . . . . . . 128

\$. 276. Epilepsie . . . . . . . . . . . . . 128

\$. 277. Tetanus . . . . . . . . . 129

\$. 278 . Chorea . . . . . . . . . . 130

\$. 279 . Hysterie . . . . . . . . . . . . . 130

§. 280. Essentielle Kinderlähmung . . . . . . . . 130

§. 281. Progressive Muskelatrophie . . . . . . . 131

9) Vergiftungen.

\$. 28\%. Vergiftungen . . . . . . . . . . . 131

§. 283. Bleivergiftung . . . . . . . . . . 131

§. 284. Delirium tremens . . . . . . . . . . . 132

10) Kranke mit Symptomen, welebe auf Erkrankung des ganzen Organismus deuten.

\$. 285. Constitutionskrankheiten . . . . . . . . 133

\$. 286. Infections- unl nicht infectiöse Krankheiten. 133

§. 287. Acute Infectionskrankheiten . . . . . . . 134

§. 288. Chronische Infectionskrankheiten . . 134

§. 289. Von Thieren übertragene Infectionskrankheiten 134

5. 290. Acute nicht infectiöse Krankheiten . . . . 131

\$. 291. Chronische nicht infectiöse Krankheiten . . . 134

1) Acute Infectionskrankheiten. . . . . 134 \$ 292. Allgemeines . . . . . . . . . . . 134 
§. 293. Pocken. . . . . . . . . . . , . 134

§. 294. Scharlach . . . . . . . . . . . . 135

§. 295. Masęrn . . . . . . . . , . . . 136

§. 296. Varicellen. . . . . . . . . . . . 136

§. 297. Febris intermittens . . . . . . . . 136

§. 298. Diphtheritis . . . . . . . . . . . 137

§. 299. Typhus recurrens. . . . . . . . , 138

§. 300. Typhus abdominalis . . . . . . . . 139

§. 301. Typhus exanthematicus . . . . . . 140

8. 302. Cholera . . . . . . . . . . . 141

§. 303. Cholera nostras . . . . . . . . . 141

§. 304. Choleratyphoid . . . . . . . . . . 142

§. 305. Dysenterie . . . . . . . . . . . 142

\$. 306. Meningitis cerebrospinalis epidemica . 142

§. 307. Septhämie. . . . . . . . . . . . 143

§. 308. Pyämie. . . . . . . . . . . . . 144

2) Chronische Infectionskrankbeiten . . 144

§. 309. Allgemeines. . . . . . . . . . . 144

§. 310. Syphilis acquisita . . . . . . . . . . . 145

§. 311. Syphilis congenita . . . . . . . . 148

3) Von Thieren auf Menschen übertragene

Infectionskrankheiten . . . . . . 148

§. 312. Trichinosis . . . . . . . . . . . 148

§. 313. Lyssa humana . . . . . . . . . . 149

4) Acute nicht infectiöse Krankheiten . 150

§. 314. Grippe (Influenza) . . . . . . . . 150

§. 315. Rheumatismus articulorum acutus. . . 151

§. 316. Acute Gicht (Arthritis) . . . . . . 151

5) Chronische wicht infectiöse Krank-

heiten . . . . . . . . . . 152

§. 317. Chlorosis . . . . . . . . . . . . 152

§. 318. Scorbut . . . . . . . . . . . . . 152

§. 319. Scrophulosis . . . . . . . . . . 153

§. 320 . Leukämie . . . . . . . . . . . . 153

\$. 321. Rhachitis . . . . . . . . . . . . 154

§. 322. Diabetes mellitus . . . . . . . . . 155

§. 323. Diabetes inositus . . . . . . . . . 156

§. 324. Diabetes insipidus . . . . . . . . 156

Alphabetisches Register . . . . . . . . . . 157 